\title{
Ultrasonic Synthetic Aperture Focusing Using the Root-Mean-Square Velocity
}

\author{
Ruey-Chyuan Shih • Young-Fo Chang • \\ Chih-Hsiung Chang • Po-Yen Tseng
}

Received: 22 February 2013 / Accepted: 29 August 2013 / Published online: 18 September 2013

(c) The Author(s) 2013. This article is published with open access at Springerlink.com

\begin{abstract}
The synthetic aperture focusing technique (SAFT) can improve the image resolution of ultrasonic testing (UT) by applying a delay-and-sum (DAS) process to the received echoes. The drawback of using current SAFT to test a multilayer medium is that the computation of delays is complicated and time-consuming. In this study, we propose a fast and simple method to calculate the approximated delays for using in SAFT to image flaws in multi-layer media without losing the resolution of the SAFT image. The approximated delays can be easily and quickly calculated by a hyperbolic time-distance relationship which is a function of the rootmean-square velocity $\left(V_{r m s}\right)$ of layers under circumstances of short lateral distance and horizontal layers. The error of approximated delays is very small when incorporating the amplitudes radiated from the transducer into the processing of SAFT. Two extreme experiments of immersion testing were carried out to test and evaluate the proposed method. The experimental results show that the proposed method is feasible.

Thus, the approximated delays calculated by the $V_{r m s}$ for using in SAFT to enhance the resolution of the scan image for testing the flaws in multi-layer medium are recommended.
\end{abstract}

Keywords SAFT · Ultrasonic testing · Root mean square velocity

R.-C. Shih · Y.-F. Chang $(\varangle) \cdot$ P.-Y. Tseng

Department of Earth and Environment Sciences, National Chung

Cheng University, Chiayi 62102, Taiwan

e-mail: seichyo@ccu.edu.tw

C.-H. Chang

General Education Center, National Chiayi University,

Chiayi 62103, Taiwan

\section{Introduction}

Ultrasonic testing (UT) is a nondestructive testing method which offers efficiency, deeper penetration and no-harm to the environment; therefore, it is commonly used to evaluate and diagnose flaws inside materials. Following the fast progress in electronic and computing technologies, digital signal processing (DSP) techniques have devoted itself to the improvement of the resolution of UT images. The synthetic aperture focusing technique (SAFT) is one such DSP technique which attempts to reform an image from a normal incident view by refocusing the received signals to where they were originally reflected and/or diffracted. With SAFT, an image of much higher resolution can be obtained. Two popular reviews of the use of SAFT in UT have been published $[1,2]$. In addition, since a higher resolution of an image is needed for precise diagnosis in medical applications, SAFT has also become more popular in medical ultrasound imaging [3-5].

The implementation of SAFT can be carried out in time or frequency domains. In a time domain, the process simply applies the delay-and-sum (DAS) technique to the received signals; this process is direct but time-consuming [1, 6] and [7]. As an alternative, the operation of SAFT can be speeded up in the frequency domain by weighting and transforming the received signals in the frequency-wavenumber domain $[8,9]$. This technique is now commonly used in SAFT for ultrasonic nondestructive testing (NDT) [10-12]. However, the manipulation of SAFT in the frequency domain requires sufficient data in time and space to cover sufficient bandwidths of frequencies and wavenumbers.

The ultrasound should refract into the specimen when it travels through the boundary between the couplant (water) and specimen in immersion testing. Therefore, the timedistance relationship of ultrasounds diffracted from a point 
reflector inside a specimen will be more complex than those with no refraction effect. However, a virtual source method still could be used to image the flaws in the specimen, and then the velocity of the longitudinal wave of the specimen could be used to implement SAFT for cases of planar pulseecho transducers [13] and focused transducers [14, 15]. Due to the phase aberration, the quality of the SAFT image obtained by the virtual source SAFT might be degraded. This will become more obvious when there exists a high velocity contrast between the couplant and specimen. Moreover, the resolution of an SAFT image could be further improved by considering the refracted wave field, such as refraction corrected SAFT $[16,17]$. However, determining delays for implementing SAFT in such cases is a difficult task.

In seismic exploration, the earth is treated as a multilayer structure and the seismic waves are refracted when they propagate across any layer-interface, which is similar to what we encounter in immersion testing (multi-layer case). The time-distance relationship of the seismic waves reflected from the multi-layer earth is approximated by a hyperbolic curve. This relationship can be fitted by an appropriate velocity called stacking velocity. The subsurface image is then revealed by correcting and stacking the reflective seismic waves based on the hyperbolic time-distance relation $[18,19]$. The above process is similar to the DAS used in SAFT. Moreover, for the horizontal strata and short offset reflections, the stacking velocity can be approximated by the root-mean-square velocity $\left(V_{r m s}\right)[20,21] . V_{r m s}$ is a function of the interval propagation times of layers and can be easily and quickly calculated.

Determining delays is the most important procedure for implementing SAFT. However, for a multi-layer medium, the computation of delays is complicated and time-consuming. Therefore, in this paper, we will use the $V_{r m s}$ to calculate the approximated delays for implementing SAFT to image flaws in multi-layer media. In the following sections, the theory of SAFT is reviewed and the $V_{r m s}$ time-distance equation of the ultrasound reflected from a multi-layer medium is derived. Two purposely designed physical experiments were conducted to test the proposed method and the performances of incorporating the $V_{r m s}$ into SAFT are evaluated. Finally, the discussions and conclusions are given.

\section{Theory}

\subsection{SAFT in a Homogeneous Medium}

When applying a single probe pulse-echo ultrasonic NDT to scan a point reflector in water, the B-scan, $i(t, x)$, shows itself as a hyperbola in the image due to spreading of the ultrasound from the transducer (Fig. 1(a)). The idea of SAFT is to form a normal incident view from the probe and refocus the hyperbolic echoes into a high energy concentration image, such as $i^{\prime}\left(t_{0}, x_{0}\right)$ in Fig. 1(b).

Since the implementation of SAFT in the time domain is simple and direct, we will first illustrate the SAFT in the time domain for a point reflector in water. Figure 2 shows that a point reflector is scanned by immersion testing. The probe is positioned at $x_{0}$, which is right above the flaw by a distance of $d$. The scan interval in the lateral direction is $\Delta x$. The two-way propagation time $\left(t_{0}\right)$ for the ultrasound to propagate from the probe to the point reflector can be expressed as

$t_{0}=\frac{2 d}{V}$,

where $V$ is the velocity of the longitudinal wave of water. If the probe is not right on the top of the point reflector, the time-distance relationship of the ultrasound then becomes

$t_{n \Delta x}=\frac{2 l}{V}=\frac{2 \sqrt{(n \Delta x)^{2}+d^{2}}}{V}, \quad n=0,1,2, \ldots$,

where $n \Delta x$ is the horizontal distance measured from $x_{0}$ to the current probe position; $l$ is the distance between the current probe position and point reflector (Fig. 2 (a)). If we square both sides of Eq. (2) and incorporate it with Eq. (1), then Eq. (2) becomes

$t_{n \Delta x}^{2}=t_{0}^{2}+\frac{(2 \cdot n \Delta x)^{2}}{V^{2}}$.

The above equation shows a hyperbolic trajectory of the time-distance relation. Then, we can find the delay $\left(\Delta t_{n}\right)$ for the neighboring time histories with lateral distance $n \Delta x$, which is

$\Delta t_{n}=t_{n \Delta x}-t_{0}=\frac{2}{V}\left(\sqrt{(n \Delta x)^{2}+d^{2}}-d\right)$.

The SAFT image thus can be obtained by summing the delayed time histories of the scan,

$i^{\prime}\left(t_{0}, x_{0}\right)=\sum_{n=0}^{m} i\left(t_{0}+\Delta t_{n}, x_{0} \pm n \Delta x\right)$,

$m \Delta x$ is the maximum lateral distance where the waves diffracted from the point reflector can be detected by the probe. Although the SAFT is based on the ultrasound diffracted from a point reflector, fortunately, an extended reflector can be thought of as a number of point sources and so SAFT work equally well in such cases. Assuming a scan environment where the vertical distance between the probe and point reflector is $1.5 \mathrm{~cm}$ and the velocity of the longitudinal wave of water is $1500 \mathrm{~m} / \mathrm{s}$ (Fig. 2(a)), the time-distance relationship of the ultrasound diffracted from the point reflector calculated by Eq. (3) is shown in Fig. 2(b). Since the 
Fig. 1 Sketch of the idea of SAFT, (a) a B-scan image for scanning a point reflector in water and (b) a normal incident view image from the probe after SAFT processing

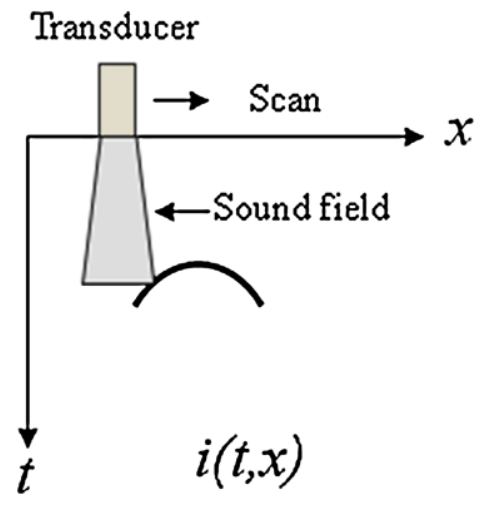

(a)
Fig. 2 Time-distance relationship of the ultrasound diffracted from a point reflector, (a) the geometry of setting and (b) the time-distance relationship; it is a hyperbola

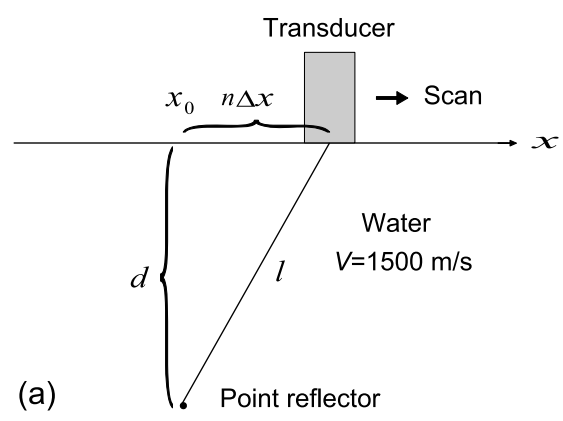

(b)

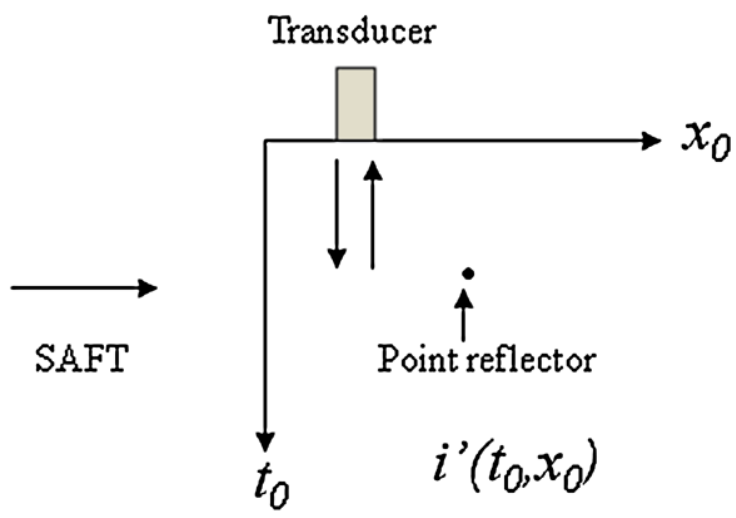

Lateral distance $(\mathrm{cm})$

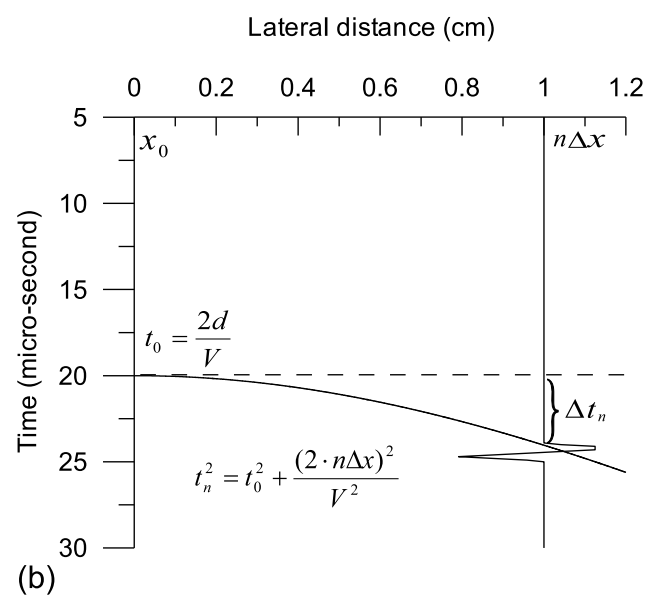

(b)

time-distance relationship is symmetrical with respect to the $x_{0}$, only the positive $n \Delta x$ is shown in Fig. 2(b).

\subsection{SAFT in a Multi-layer Medium}

Since the velocity and density of water are much lower than those of the solid specimens tested in ultrasonic NDT, the refraction effect will be stronger for the ultrasound propagating through the water-specimen interface. Therefore, the water-specimen medium is an extreme case of two-layer media for the refraction effect. This provides a good opportunity to evaluate the proposed method.

While scanning a point reflector inside a specimen during immersion testing, the ultrasound is refracted when propagating through the water-specimen interface. In this case, the vertical two-way propagation time should be expressed as

$t_{0}=\frac{2 d_{1}}{V_{1}}+\frac{2 d_{2}}{V_{2}}$

where, $d_{1}$ is the vertical distance between the probe and the specimen, $d_{2}$ is the depth of the point reflector measured from the water-specimen interface, and $V_{1}$ and $V_{2}$ are the velocities of the longitudinal wave of water and specimen (Fig. 3(a)). When the probe is moved to a scan position at distance $n \Delta x$ from $x_{0}$, the two-way propagation time can be expressed as

$t_{n \Delta x}=\frac{2 l_{1}}{V_{1}}+\frac{2 l_{2}}{V_{2}}$,

where $l_{1}$ and $l_{2}$ are the ray paths in water and the specimen, respectively (Fig. 3(a)). It may be cumbersome to determine the true root from the four roots by solving the Eq. (7) (a quartic equation) directly. Therefore, the commonly used method for obtaining $t_{n}$ is the trial-and-error ray tracing method which shoots out dense rays from the probe and finds out which ray is closest to the target within the acceptable error as the ray of the target shot. Hereafter, in this study, the propagation time calculated by the trial-anderror ray tracing method is considered as the true propagation time which its accuracy is within 0.001 microsecond in order to fit the requirement of this study. Moreover, the true delay is taken as the difference between the true propagation time and vertical propagation time. However, the trial-and-error ray tracing method is complicated and timeconsuming for the two-layer medium. For more than two layers cases, the difficulty and computation time increase dramatically. 


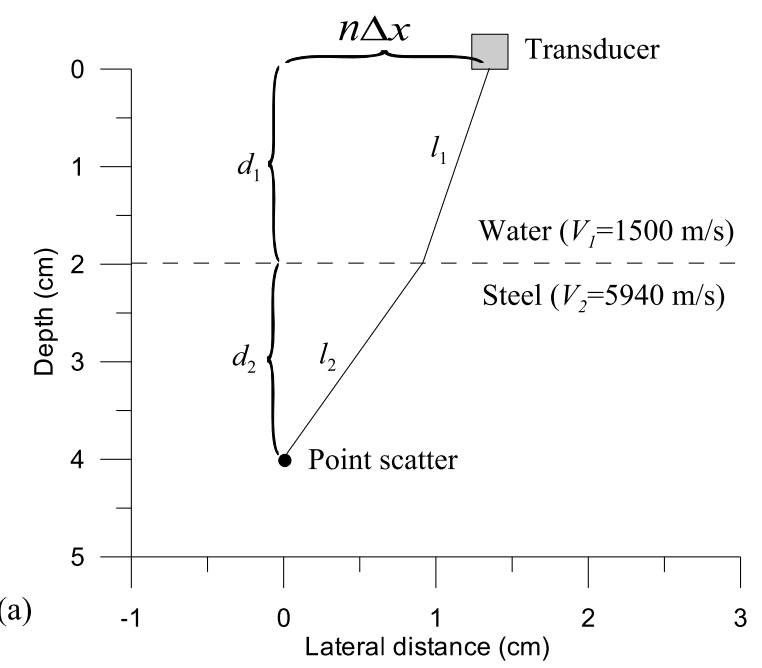

Fig. 3 (a) Geometry of a point reflector in a steel specimen for immersion testing, (b) the propagation time of the ultrasound diffracted from the point reflector along the lateral distance, where the black line stands for the true propagation time, and the blue and red lines are those calcu-

To generalize to a continuous time-distance relationship, similar to Eq. (3), the squared reflection time of ultrasound is function of the squared lateral distance [20-22]

$t_{x}^{2}=f\left(x^{2}\right)$.

Taking $x^{2}$ as the independent variable, the squared reflection time in the form of a Maclaurin power series (Taylor series expansion at $x=0$ ) becomes [20]

$t_{x}^{2} \approx t_{0}^{2}+\left.\frac{x^{2}}{1 !} \frac{d}{d x^{2}} f\left(x^{2}\right)\right|_{x=0}+\left.\frac{x^{4}}{2 !} \frac{d^{2}}{d x^{4}} f\left(x^{2}\right)\right|_{x=0}+\cdots$

Neglecting the third and higher order terms, Eq. (9) becomes

$t_{x}^{2}=t_{0}^{2}+\left.x^{2} \frac{d}{d x^{2}} f\left(x^{2}\right)\right|_{x=0}$.

However $\left.\frac{d}{d x^{2}} f\left(x^{2}\right)\right|_{x=0}=\frac{2^{2}}{V_{r m s}^{2}}$, where $V_{r m s}$ is the rootmean-square velocity [20, 21].

Then, Eq. (10) becomes

$t_{x}^{2}=t_{0}^{2}+\frac{(2 x)^{2}}{V_{r m s}^{2}}$.

For an $N$-layer medium, $V_{r m s}$ is defined as

$V_{r m s}^{2}=\frac{V_{1}^{2} t_{I 1}+V_{2}^{2} t_{I 2}+V_{3}^{2} t_{I 3}+\cdots+V_{N}^{2} t_{I N}}{t_{I 1}+t_{I 2}+t_{I 3}+\cdots+t_{I N}}$.

Where $V_{1}, V_{2}, V_{3}, \ldots$, and $V_{N}$, are the velocities of the longitudinal wave of layers from 1 to $N . t_{I 1}, t_{I 2}, t_{I 3}, \ldots$, and

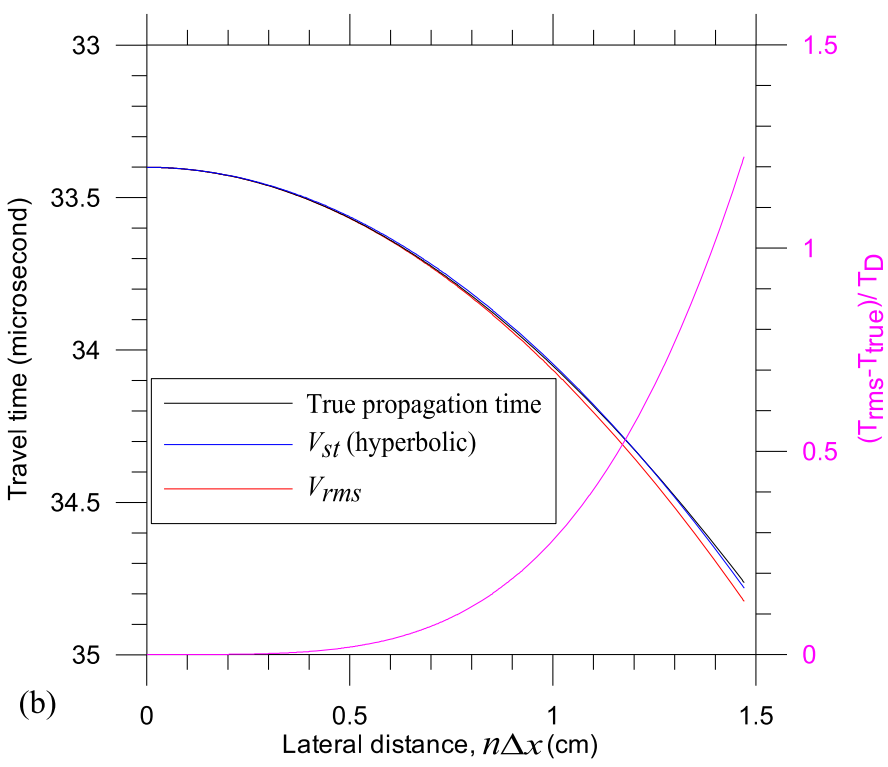

lated by $V_{s t}$ and $V_{r m s} . T_{D}$ is the dominant period of the ultrasound used in this study, and it is 0.05 microsecond (or the frequency is $20 \mathrm{MHz}$ ). The pink line is the ratio of the propagation time error computed by the $V_{r m s}$ to $T_{D}$

$t_{I N}$ are their one-way interval propagation time, for examples $t_{I 1}=d_{1} / V_{1}$ and $t_{I 2}=d_{2} / V_{2}$. For the discrete lateral distance, Eq. (11) becomes

$t_{n \Delta x}^{2}=t_{0}^{2}+\frac{(2 \cdot n \Delta x)^{2}}{V_{r m s}^{2}}$.

The best fitting time-distance relationship of the reflection ultrasound by a hyperbolic equation is [20,21]

$t_{n \Delta x}^{2}=t_{0}^{2}+\frac{(2 \cdot n \Delta x)^{2}}{V_{s t}^{2}}$

where $V_{s t}$ is the stacking velocity computed by fitting the time-distance relationship of the reflection ultrasound. $V_{r m s}$ is generally smaller than $V_{s t}\left(V_{r m s} \leq V_{s t}\right)$ [20] and it is a function of vertical propagation time. In ultrasonic NDT, the beam width of the sound field radiating from the transducer is usually small (smaller than 15 degrees), which follows the assumption (Maclaurin series expansion) of a very short lateral distance. Therefore, the approximated delays can be easily calculated by Eq. (12) and Eq. (13) and used in SAFT.

The error of using $V_{r m s}$ to calculate the propagation time of the ultrasound diffracted from a point reflector is estimated. Assuming the distance between the probe and specimen is $2 \mathrm{~cm}$ and the depth of the point reflector is $2 \mathrm{~cm}$ below the water-specimen interface, the velocities of the longitudinal wave of water and specimen are $1500 \mathrm{~m} / \mathrm{s}$ and $5940 \mathrm{~m} / \mathrm{s}$ (Fig. 3(a)), respectively. In Fig. 3b, the black line stands for the true propagation time of the ultrasound 


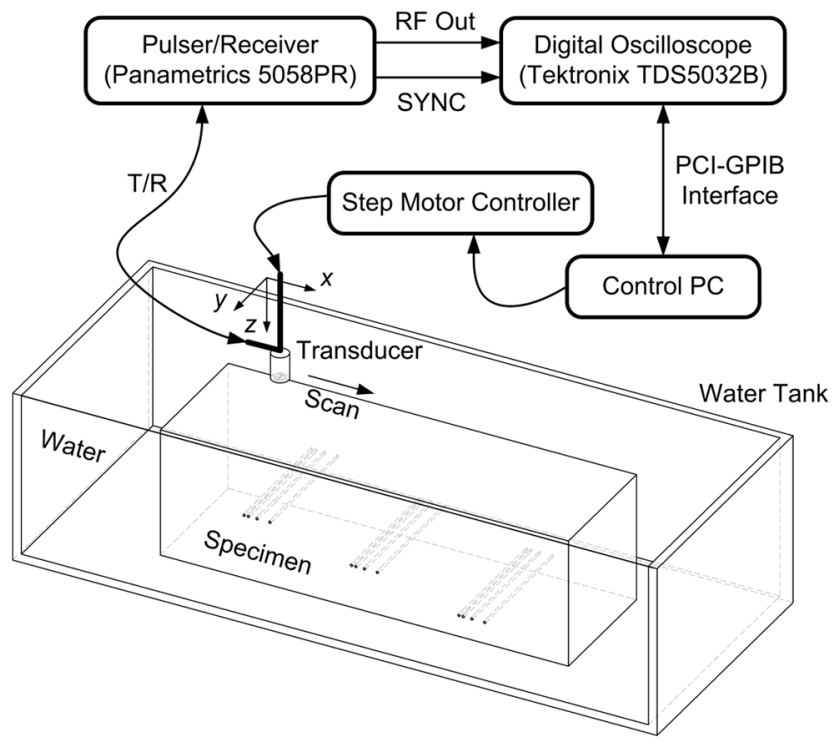

Fig. 4 Block diagram of the automatic immersion scanning system

diffracted from the point reflector, and the blue and red lines are those calculated by the $V_{s t}$ and $V_{r m s}$ (Fig. 3(b)), respectively. The three lines are nearly coincident at a short lateral distance, i.e. $n \Delta x<0.5 \mathrm{~cm}$, where the incident angle of the ray is 2.8 degrees and the ratio of lateral distance to the flaw depth $\left(d_{1}+d_{2}\right)$ is 1:8. As the lateral distance increases, the propagation time of the ultrasound computed by $V_{r m s}$ begins to deviate from the other two and the error of propagation time is 0.05 microsecond at lateral distance of $1.4 \mathrm{~cm}$, where the incident angle of the ray is 7.2 degrees and the ratio of lateral distance to the flaw depth is 1.4:4. The dominant period $\left(T_{D}\right)$ of the ultrasound used in this study is 0.05 microsecond (or the frequency is $20 \mathrm{MHz}$ ). The pink line in Fig. 3(b) shows the ratio of the propagation time error computed by the $V_{r m s}$ to $T_{D}$, and the ratios at lateral distances 0.5 and $1.4 \mathrm{~cm}$ are 0.018 and 1 , respectively. The beam width of the transducer used in this study is about 6 degrees; the half angle of the beam width is only 3 degrees. As we can see from these results, the error of the approximated propagation time is very small.

\section{Experiments and Results}

To validate the proposed algorithm, we designed and carried out two physical experiments of immersion testing. A schematic diagram of the data acquisition system of our experiments is shown in Fig. 4. A pulse-receiver (Panametrics 5058PR) in pulse echo mode (T/R) was used to excite the transducer and synchronize the digital oscilloscope. A Tektronix TDS-5032B digital oscilloscope received and digitized the signal with a sampling interval of $8 \times 10^{-9}$ second. A personal computer (PC) retrieved the digitized radio frequency (RF) signal from the oscilloscope via IEEE-488 communications (GPIB). A step motor driven by the PC was used to move the probe automatically as well. A $20 \mathrm{MHz}$ immersion transducer (Panametrics V316-SU) of $3 \mathrm{~mm}$ in diameter transmitted and received the ultrasound. Two steel (SKD61) specimens with $5940 \mathrm{~m} / \mathrm{s}$ in longitudinal wave velocity and $7.69 \times 10^{-3} \mathrm{~kg} / \mathrm{cm}^{3}$ in density were fabricated and scanned. Water served as the couplant. The distance between the probe and specimen was $20 \mathrm{~mm}$ and the scan interval was $0.5 \mathrm{~mm}$ laterally.

\subsection{Testing for Three Single-Side-Drilled Holes}

A steel specimen with three single-side-drilled holes located at different depths was fabricated (Fig. 5(a)). The thickness and width of the specimen are both $30 \mathrm{~mm}$. Diameter of holes is $0.5 \mathrm{~mm}$, and depths of centers of holes are $25 \mathrm{~mm}$, $20 \mathrm{~mm}$ and $15 \mathrm{~mm}$, respectively from left to right. These single-side-drilled holes are treated as the point flaws. Figure 5 (b) shows a B-scan image of the steel specimen. The abscissa of the image is the lateral position of the scan and the ordinate is the depth measured from the top surface of the specimen. All the images in this study are displayed by a gray scale, black and white express the maximum and zero amplitudes of the echoes and the change from black to white is linear. Three curve events with apexes positioned at depths of $25 \mathrm{~mm}, 20 \mathrm{~mm}$ and $15 \mathrm{~mm}$ were imaged from the three single-side-drilled holes. Figure 5(c) is the SAFT image processed by the true delays. The three flaw images at different depths have been successfully synthesized with $1 \mathrm{~mm}$ horizontal size. Figure 5(d) is the SAFT image processed by the approximated delays. Since the delays used in SAFT are approximated values, the intensities of flaw images (Fig. 5(d)) are weaker than those in Fig. 5(c). Nonetheless, both SAFT images are alike.

The array performance indicator (API) [23] is a useful index to quantify the performances of the two methods, which measured the area $\left(A_{-6 \mathrm{~dB}}\right)$ of the flaw image where the image intensity is within $-6 \mathrm{~dB}$ of its peak intensity and normalized by the square of the bulk longitudinal wavelength. The $A_{-6} \mathrm{~dB}$ areas of flaw images of the three different depths flaws and their API values for the two methods are shown in Fig. 6. The average ratio of the API values of the two methods $\left(\overline{A P I}_{\text {true/app. }}\right)$ is 0.94 . Figure 7 shows the normalized amplitudes of SAFT images (Fig. 5(c) and (d)) along axial and lateral directions to the same scale. The red and blue amplitudes are the reconstructed images processed by the true delay SAFT and approximated delay SAFT. In Fig. 7(a), the normalized amplitudes of images run right through centers of the hole-flaws in axial direction. A higher noise level can be seen by using the proposed method. Furthermore, Fig. 7(b) shows the normalized amplitudes of images in lateral direction. Depths of normalized amplitudes of images 
Fig. 5 SAFT image of immersion testing for three single-side-drilled holes located at different depths in a steel specimen, (a) the configuration of the specimen, (b) the B-scan image, (c) the SAFT image processed by the true delays and (d) the SAFT image processed by the approximated delays
Transducer

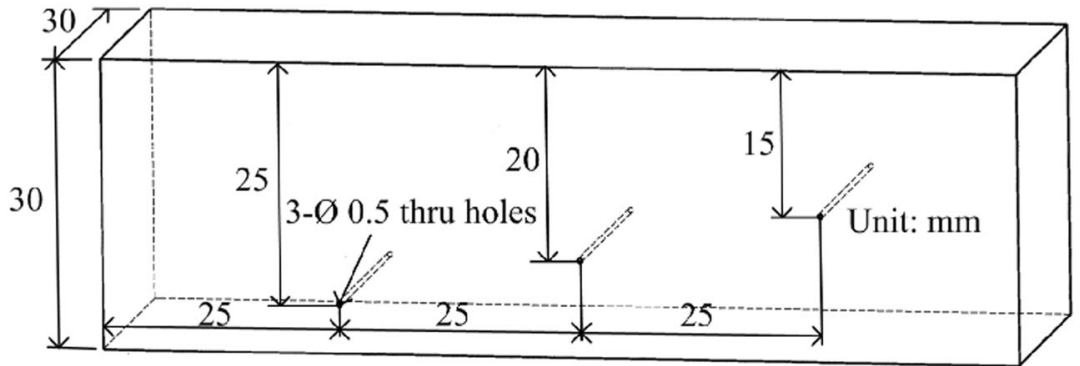

(a)

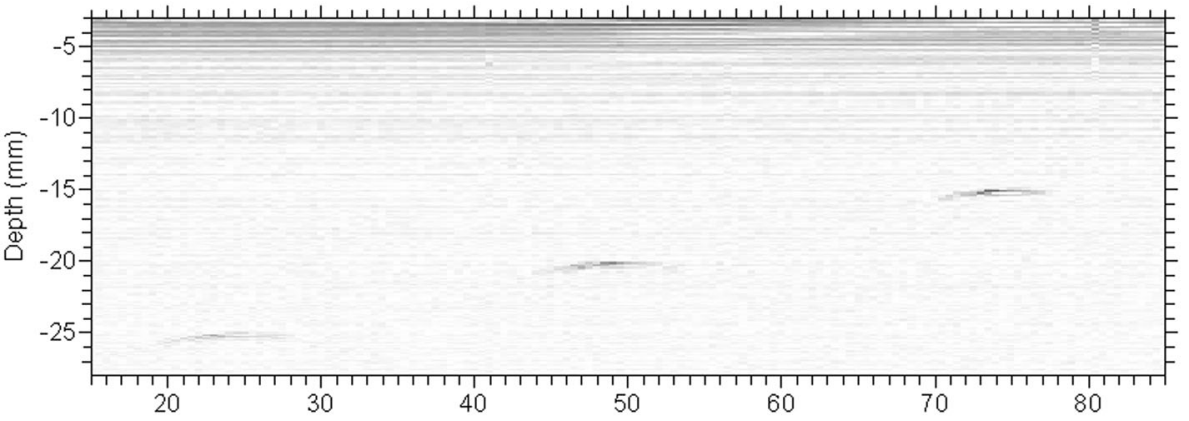

(b)

Lateral distance $(\mathrm{mm}$ )

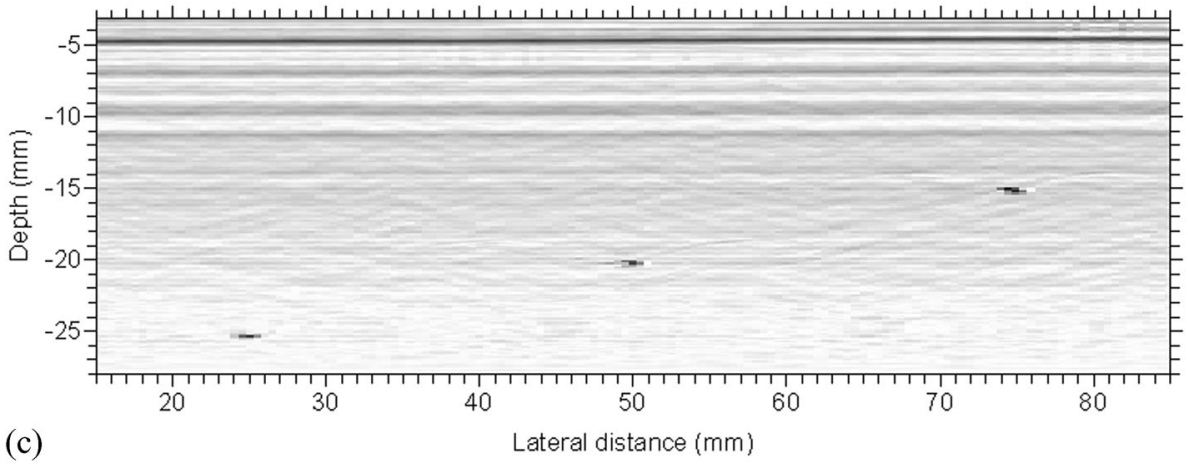

(d)

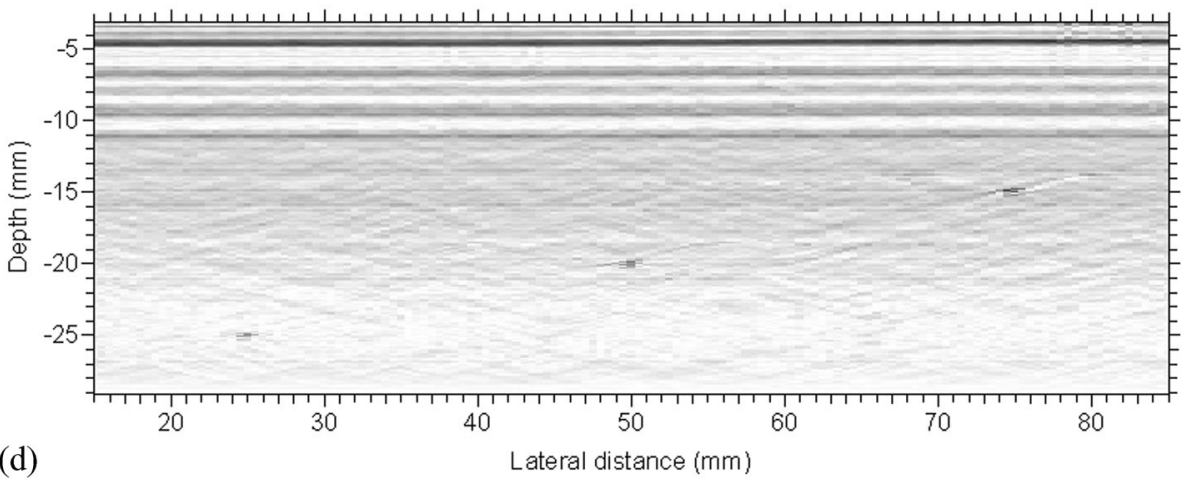

are at $25 \mathrm{~mm}, 20 \mathrm{~mm}$ and $15 \mathrm{~mm}$, from left to right, respectively. The lateral resolutions of images are almost the same for both SAFT methods. Therefore, based on Figs. 6 and 7, our simple and fast calculation method for approximated delays used in SAFT is feasible.

\subsection{Testing for Three Quad-Side-Drilled Holes}

To test how the resolution is deteriorated by using the approximated delays for SAFT, a steel specimen was made with three sets of quad-side-drilled holes located at differ- 
ent depths. The dimension of holes is the same as those used in the previous specimen (Fig. 5(a)). For each quad-sidedrilled set, there are four holes and the horizontal intervals between the holes are $3 \mathrm{~mm}, 2 \mathrm{~mm}$ and $1 \mathrm{~mm}$, respectively, from left to right (Fig. 8(a)). Depths of the three sets are settled at $25 \mathrm{~mm}, 20 \mathrm{~mm}$ and $15 \mathrm{~mm}$. The B-scan image of the specimen is shown in Fig. 8(b). As we can see from the image, the holes in the sets were not resolved. The image was
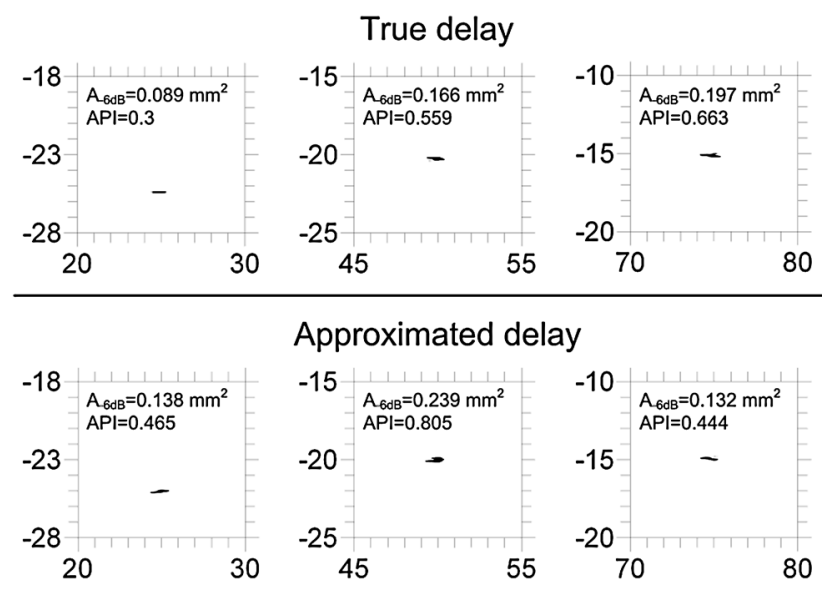

Fig. 6 Images of $-6 \mathrm{~dB}$ areas $\left(A_{-6 \mathrm{~dB}}\right)$ of the three different depths flaws and their API values for the true and approximated delays SAFT methods then processed by the SAFT with the true and approximated delays, respectively; the results are demonstrated in Fig. 8(c) and 8(d). As seen Fig. 8(c) and 8(d), the holes with $3 \mathrm{~mm}$ and $2 \mathrm{~mm}$ in between them can be easily identified. However, this is not the case for those with $1 \mathrm{~mm}$ horizontal intervals in the different depth quad-side-drilled holes sets. Again, the reconstructed images of holes illustrated in Fig. 8(d) show weaker intensity than those demonstrated in Fig. 8(c), but both SAFT images are alike.

The $A_{-6 \mathrm{~dB}}$ areas of flaw images of the three different depths quad-side-drilled holes sets and their API values for the two methods are shown in Fig. 9. Figures 9(a), 9(b) and 9 (c) are the images of holes sets at depths $25 \mathrm{~mm}$, $20 \mathrm{~mm}$ and $15 \mathrm{~mm}$, respectively. The columns of figures in Fig. 9 from left to right are the images of left, left second and right two holes of quad-side-drilled holes. The $\overline{A P I}_{\text {true/app. }}$ is 1.13 . Figure 10 shows the normalized amplitudes of SAFT images (Fig. 8(c) and (d)) along axial and lateral directions. The red and blue amplitudes are the images processed by the true delay SAFT and approximated delay SAFT, respectively. In Fig. 10(a), the normalized amplitudes of images run through the lateral distances at $25 \mathrm{~mm}, 50 \mathrm{~mm}$ and $75 \mathrm{~mm}$ in axial direction. Again, in the figure, the noise levels are higher by using the approximated delay SAFT. Figure 10(b) shows the normalized am-
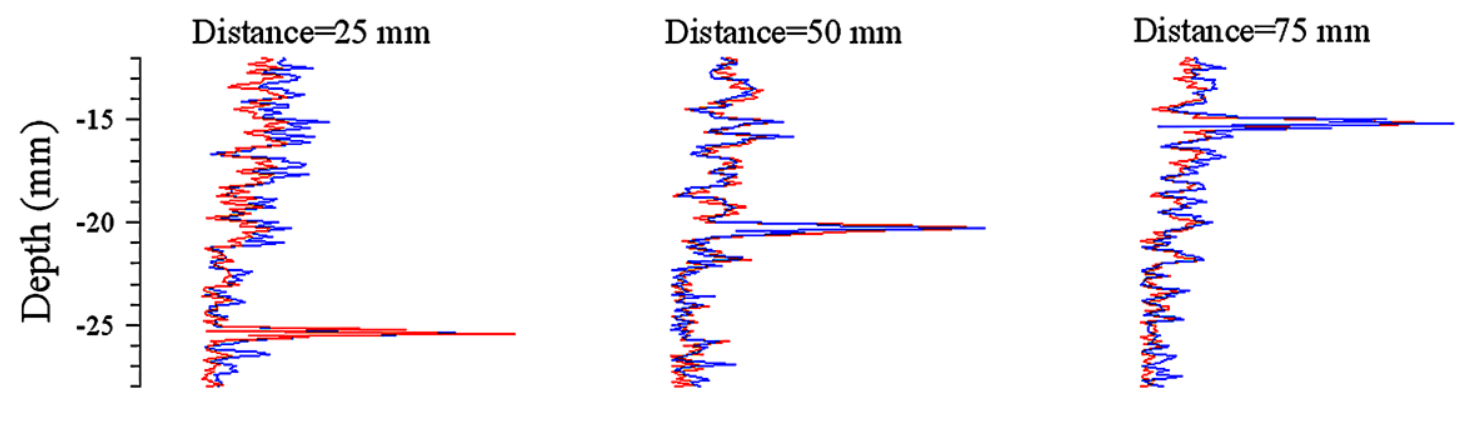

(a)

(b)

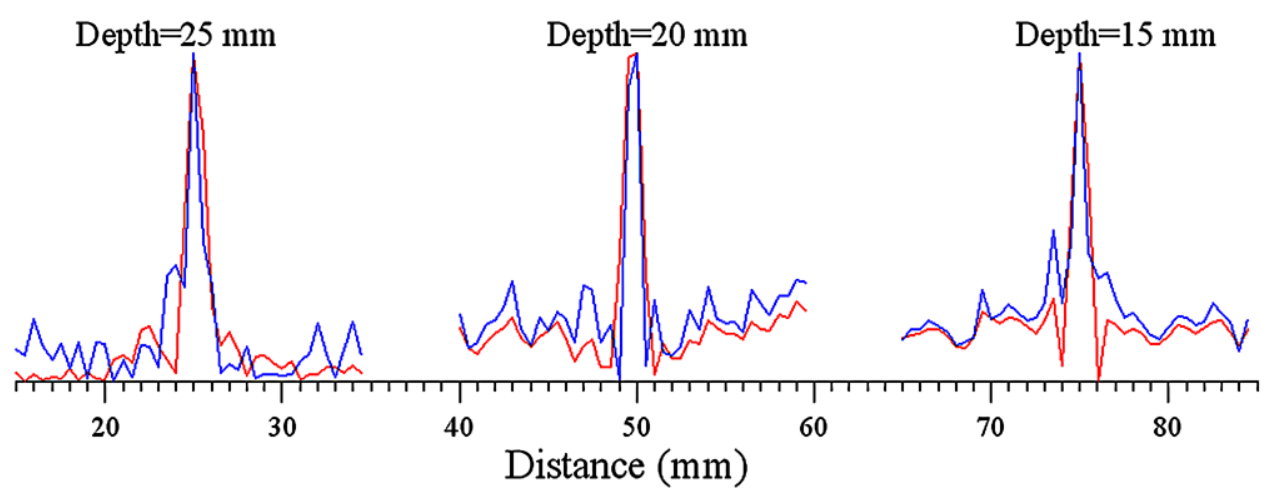

Fig. 7 The normalized amplitudes of SAFT images (Fig. 5(c) and (d)) along axial and lateral directions to the same scale, reconstructed images processed by the true velocity SAFT (red) and approximated velocity SAFT (blue). (a) The axial normalized amplitudes of flaw im- ages, lateral distances from left to right are $25 \mathrm{~mm}, 50 \mathrm{~mm}$ and $75 \mathrm{~mm}$. (b) The lateral normalized amplitudes of flaw images, depths from left to right are $25 \mathrm{~mm}, 20 \mathrm{~mm}$ and $15 \mathrm{~mm}$ 
Fig. 8 SAFT image of immersion testing for three quad-side-drilled holes located at different depths in a steel specimen, (a) the configuration of the specimen, (b) the B-scan image, (c) the SAFT image processed by the true delays and (d) the SAFT image processed by the approximated delays
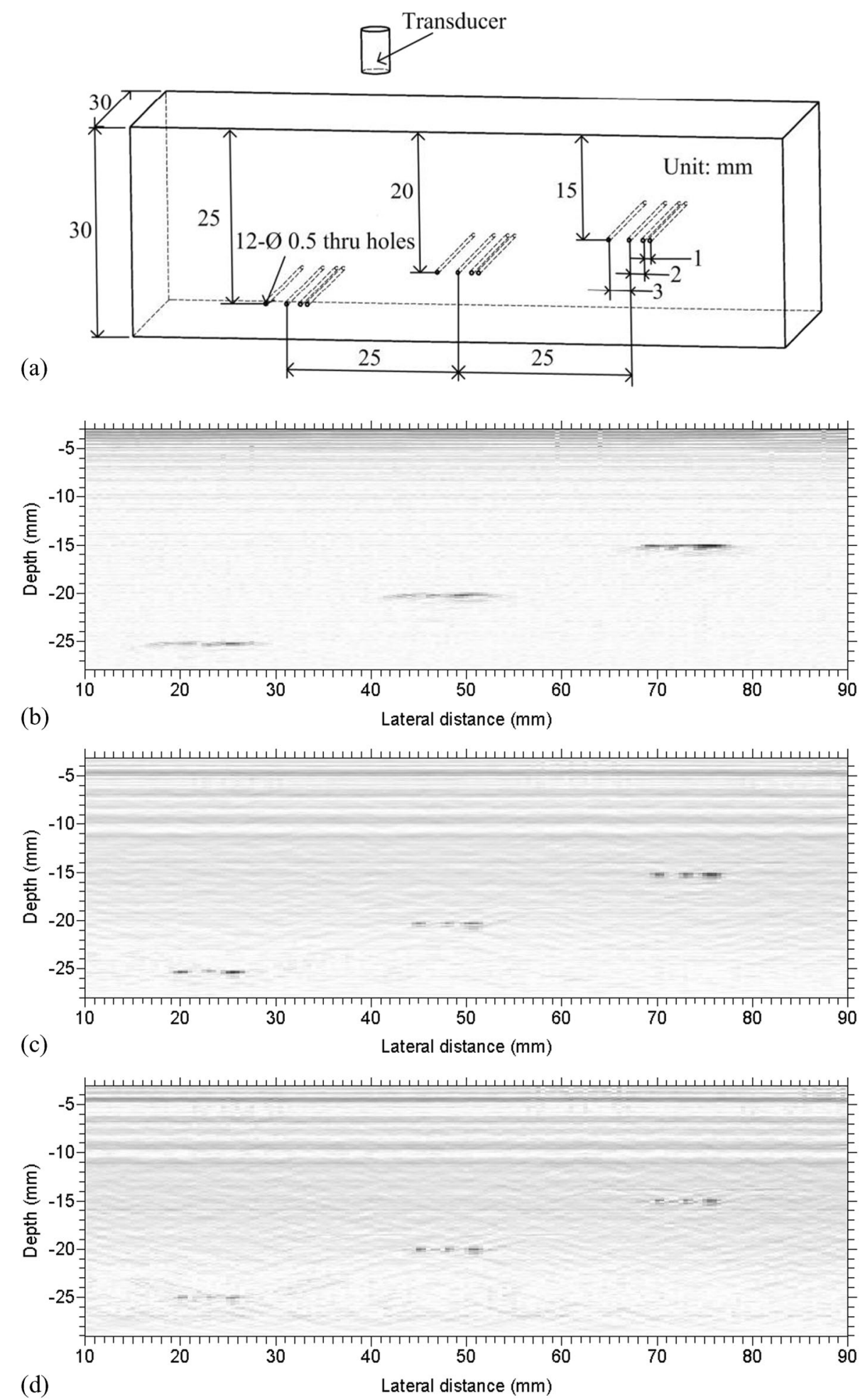

plitudes of images in lateral direction. Depths of normalized amplitudes of images are at $25 \mathrm{~mm}, 20 \mathrm{~mm}$ and $15 \mathrm{~mm}$, from left to right, respectively. The lateral resolutions of both SAFT images are almost the same. Based on Figs. 9 and 10, the resolutions of both SAFT images are almost the same.

\section{Discussions}

Point source is the common assumption of SAFT used in modern ultrasonic NDT. However, the above assumption is only true when the propagation distance of the ultrasound is greater than the size of probe. Therefore, better perfor- 
Fig. 9 Images of $-6 \mathrm{~dB}$ areas $\left(A_{-6 \mathrm{~dB}}\right)$ of the three different depths quad-side-drilled holes sets and their API values for the true and approximated delays SAFT methods. Images of holes located at depth (a) $25 \mathrm{~mm}$,

(b) $20 \mathrm{~mm}$ and (c) $15 \mathrm{~mm}$, respectively. The columns of figures from left to right are the images of left, left second and right two holes of quad-side-drilled holes

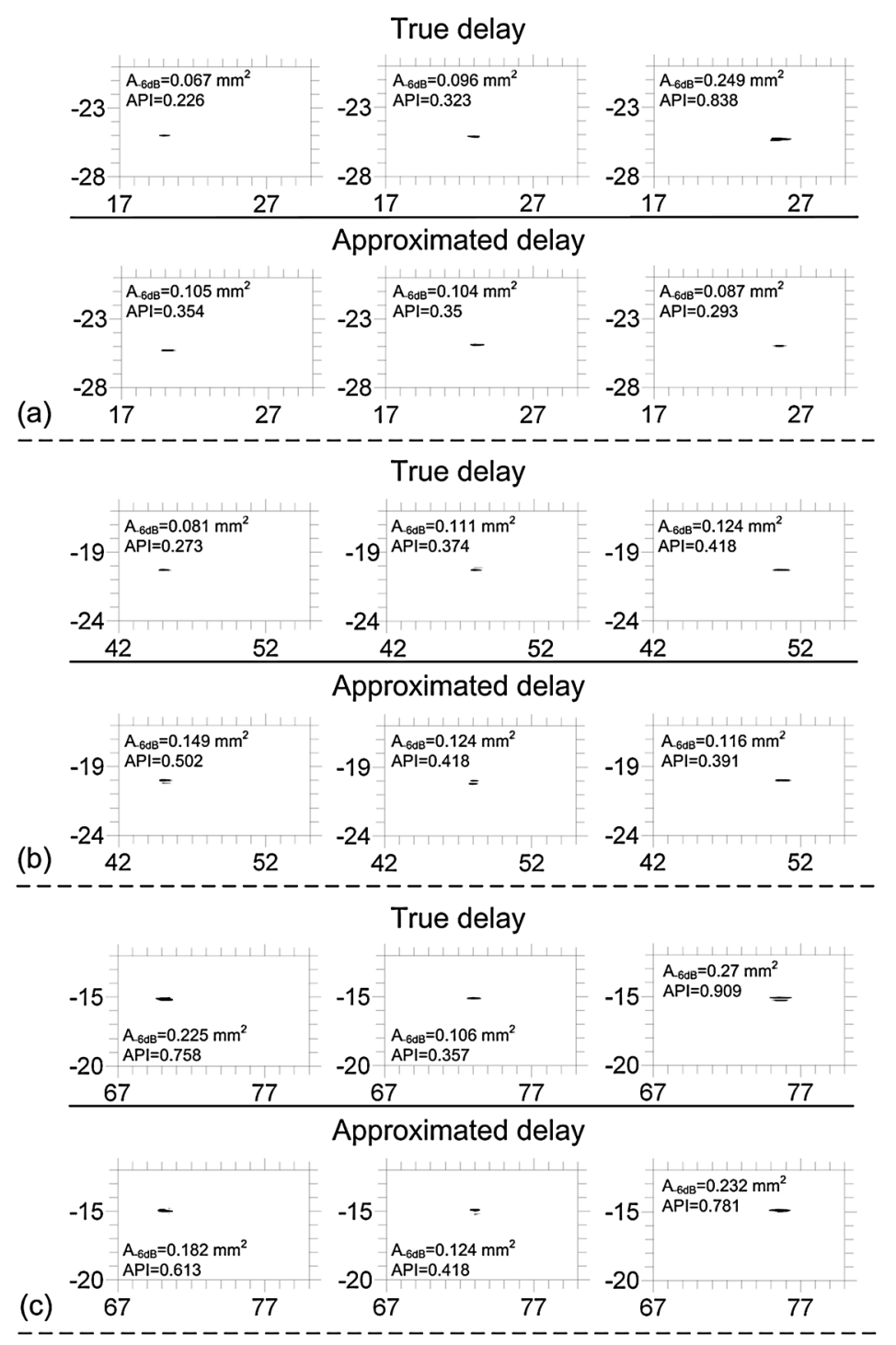

mance in detecting deep flaws by SAFT could be expected compared with those of shallow flaws. For a small size transducer, the difference in the propagation times of the ultrasound radiated from the center and rim of the probe is minor; therefore, the delays can be accurately estimated. In our experiment, the shortest distance between the probe and flaw is $35 \mathrm{~mm}$, which is about 12 times greater than the diameter of the transducer. Thus, the probe used in this study can be considered as a point source for SAFT.

Since SAFT is based on the ultrasound diffracted from a point reflector, the dimension of the flaw must be smaller than the wavelength of the ultrasound to obtain better SAFT performance. In our laboratory work, the diameter of the side-drilled hole is $0.5 \mathrm{~mm}$ and the wavelength of the ultrasound is $0.3 \mathrm{~mm}$. The ratio of the wavelength of the ultrasound to the dimension of flaw is 0.6 , which may not be suitable for an assumption of point diffraction. For the case of shallow side-drilled holes, the detected ultrasonic echoes would behave more like the reflection waves reflected from the top curve area of the side-drilled hole. This would complicate propagation time and waveform of the received echoes. On the other hand, for the deep side-drilled holes, only the ultrasound reflected from the apex of the side-drilled hole can be detected by the probe, which is like a point diffraction effect. Therefore, when using ultrasonic SAFT to image a flaw whose size is about the same as the wavelength of the ultrasound, only the detected echoes with a long propagation distance would have a better result.

The error of propagation times of the ultrasound calculated by the $V_{r m s}$ depends on the lateral distance and the velocity contrast of layers. For a small lateral distance condition, which is a common truth in ultrasonic NDT, the larger the velocity contrast of layers has, the larger the error of the propagation times is produced when using the $V_{r m s}$. However, in our case study, the velocity contrast is malevolently set up as $0.75,\left(V_{\text {steel }}-V_{\text {water }}\right) / V_{\text {steel }}$, which is very large. 

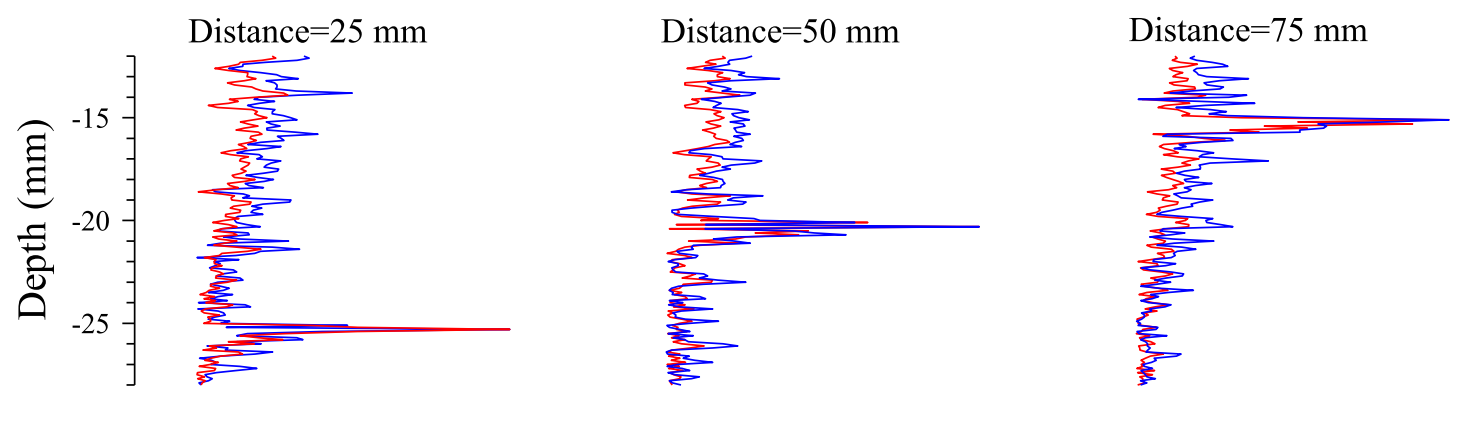

(a)

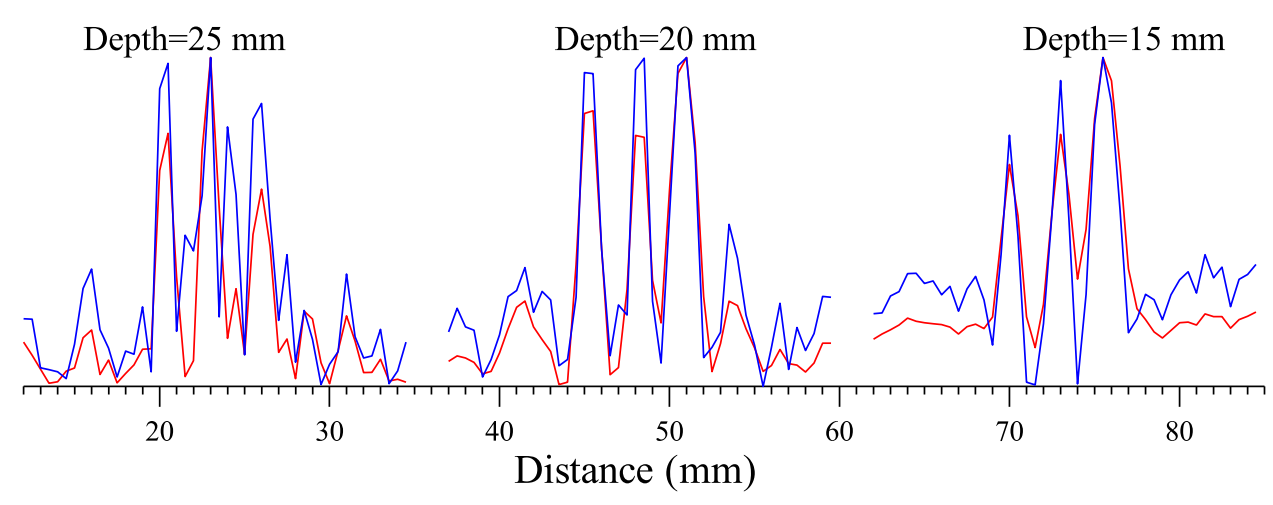

Fig. 10 The normalized amplitudes of SAFT images (Fig. 8(c) and (d)) along axial and lateral directions. The red and blue lines are the images processed by the true delay SAFT and approximated delay SAFT. (a) The axial normalized amplitudes of flaw images, lateral

However, the propagation time error is still small. Therefore, the success of using the $V_{r m s}$ of layers with large velocity contrast to implement SAFT is verified.

In addition to the error of the propagation time when using the $V_{r m s}$ to calculate the approximated delays, the amplitudes of the refracted waves and the radiation pattern of the transducer must also be taken into consideration in the practical application of SAFT. The amplitude of the refracted wave depends on the refraction coefficient of the waterspecimen interface and the incident angle of the ray. The refracted wave has the largest amplitude with a normal incident angle but the refracted amplitudes will be small for the waves with larger incident angles. The half angle of the beam width of the transducer used in this study is 3 degrees. With such a small variation of incident angles of the ultrasound, the amplitudes of the refracted waves with different incident angles are almost the same. However, the amplitudes of the ultrasound radiated from the transducer differ more. The amplitude of the ultrasound is maximum at the normal direction (for example 0 degree of incident angle), but the amplitude decreases $6 \mathrm{~dB}$ at the half angle of the beam width and it decreases sharply to zero when the radiated wave is outside the beam width. Though the waves with larger incident angles will have larger propagation time error of the ultrasound calculated by the $V_{r m s}$, their contributions distances from left to right are $25 \mathrm{~mm}, 50 \mathrm{~mm}$ and $75 \mathrm{~mm}$. (b) The lateral normalized amplitudes of flaw images, depths from left to right are $25 \mathrm{~mm}, 20 \mathrm{~mm}$ and $15 \mathrm{~mm}$

to forming the reconstructed SAFT image are weakened by their weak amplitude. Thus, using the $V_{r m s}$ to implement SAFT results in good performance.

For a multi-layer medium, the computer time for calculation of the true propagation time of the ultrasound diffracted from a point flaw will increase dramatically with the number of layers, not to mention 3-D cases. However, the computer time for calculation of the approximated delays by the $V_{r m s}$ for the multi-layer SAFT will be almost the same as that of the homogeneous medium. Therefore, efficiency must be emphasized when using the $V_{r m s}$ to implement SAFT for imaging flaws in a multi-layer medium.

\section{Conclusions}

The computation of delays is complicated and time-consuming when using SAFT to image flaws in a multi-layer medium. However, under a condition of short lateral distance and horizontal layers, the propagation time of the ultrasound diffracted from a point reflector in a multi-layer medium can be approximated by a hyperbolic time-distance relationship which is function of the $V_{r m s}$. Therefore, the approximated delays can be easily and quickly obtained for SAFT. 
The error of approximated delays calculated by the $V_{r m s}$ depends on the lateral distance and the velocity contrast of layers. The condition of short lateral distance can usually be achieved by the narrow beam widths of transducers used in ultrasonic NDT. A case of large velocity contrast of layers shows that the error of approximated delays is very small. In addition, the contribution of the travel time error of the approximated delays will be further reduced by incorporating the amplitudes radiated from the transducer into the processing of SAFT. Two extreme physical experiments of immersion testing were carried out to test and evaluate the proposed method. The experimental results show that the proposed method is successful, the resolution of SAFT image processed by the approximated delays is almost the same as those using true delays. Therefore, the proposed approach offers a simple and fast method for calculating the approximated delays by the $V_{r m s}$ for SAFT to test multi-layer media.

Open Access This article is distributed under the terms of the Creative Commons Attribution License which permits any use, distribution, and reproduction in any medium, provided the original author(s) and the source are credited.

\section{References}

1. Doctor, S.R., Hall, T.E., Reid, L.D.: SAFT-the evolution of a signal processing technology for ultrasonic testing. NDT Int. 19, 163-167 (1986)

2. Schmitz, V., Muller, W., Schafer, G.: Synthetic aperture focusing technique: state of the art. Acoust. Imag. 19, 545-551 (1992)

3. Passmann, C., Ermert, H.: A 100-MHz ultrasound imaging system for dermatologic and ophthalmologic diagnostics. IEEE Trans. Ultrason. Ferroelectr. Freq. Control 43, 545-552 (1996)

4. O’Donnell, M., Eberle, M.J., Stephens, D.N., Litzza, J.L., Vicente, K.S., Shapo, B.M.: Synthetic phased arrays for intraluminal imaging of coronary arteries. IEEE Trans. Ultrason. Ferroelectr. Freq. Control 44, 714-721 (1997)

5. Jensen, J.A., Nikolov, S.I., Gammelmark, K.L., Pedersen, M.H.: Synthetic aperture ultrasound imaging. Ultrasonics 44, e5-e15 (2006)
6. Ozaki, Y., Sumitani, H., Tomoda, T., Tanaka, M.: A new system for real-time synthetic aperture ultrasonic imaging. IEEE Trans. Ultrason. Ferroelectr. Freq. Control 35, 828-838 (1988)

7. Jensen, J.A., Nikolov, S.I., Gammelmark, K.L., Pedersen, M.H.: Synthetic aperture ultrasound imaging. Ultrasonics 44, e5-e15 (2006)

8. Chun, J.H., Jacewitz, C.A.: Fundamentals of frequency domain migration. Geophysics 46, 717-733 (1981)

9. Chang, Y.F., Chern, C.C.: Frequency-wavenumber migration of ultrasonic data. J. Nondestruct. Eval. 19, 1-10 (2000)

10. Langenberg, K.J., Berger, M., Kreutter, T., Mayer, K., Schmitz, V.: Synthetic aperture focusing technique signal processing. NDT Int. 19, 177-189 (1986)

11. Mayer, K., Marklein, R., Langenberg, K.J., Kreutter, T.: Threedimensional imaging system based on Fourier transform synthetic aperture focusing technique. Ultrasonics 28, 241-255 (1990)

12. Stepinski, T.: An implementation of synthetic aperture focusing technique in frequency domain. IEEE Trans. Ultrason. Ferroelectr. Freq. Control 54, 1399-1408 (2007)

13. Burch, S.F., Burton, J.T.: Ultrasonic synthetic aperture focusing using planar pulse-echo transducers. Ultrasonics 22, 275-281 (1984)

14. Rupitsch, S.J., Zagar, B.G.: Acoustic microscopy technique to precisely locate layer delamination. IEEE Trans. Instrum. Meas. 56, 1429-1434 (2007)

15. Chang, C.H., Chang, Y.F., Ma, Y., Shung, K.K.: Reliable estimation of virtual source position for SAFT imaging. IEEE Trans. Ultrason. Ferroelectr. Freq. Control 60, 356-363 (2013)

16. Chang, Y.F., Wu, T.Y.: Angle beam shear-wave nondestructive testing. Mater. Eval. 63, 827-831 (2005)

17. Chang, Y.F., Hsu, H.I.: Ultrasonic synthetic aperture imaging for correcting the refraction artifacts applied to angle beam immersion testing. J. Test. Eval. 34, 1-5 (2006)

18. Dobrin, M.B., Savit, C.H.: Introduction to Geophysical Prospecting, pp. 239-242. McGraw-Hill, New York (1988)

19. Yilmaz, O.: Seismic Data Processing, pp. 274-283. Society of Exploration Geophysicists, Tulsa (1987)

20. Kleyn, A.H.: Seismic Reflection Interpretation, pp. 73-76. Elsevier Applied Science Publishers, New York (1983)

21. Taner, M.T., Koehler, F.: Velocity spectra-digital computer derivation applications of velocity functions. Geophysics 34, 859881 (1969)

22. Green, C.H.: Velocity determinations by means of reflection profiles. Geophysics 3, 295-305 (1938)

23. Holmes, C., Drinkwater, B.W., Wilcox, P.D.: Post-processing of the full matrix of ultrasonic transmit-receive array data for nondestructive evaluation. NDT Int. 38, 701-711 (2005) 